\title{
DITERPENOS DE NÚCLEO KAURANO COMO INHIBIDORES DE LA PTR1 DE LEISHMANIA: UN ESTUDIO in silico
}

\section{KAURANE-RELATED DITERPENES AS LEISHMANIA PTERIDINE REDUTASE INHIBITORS: AN IN SILICO STUDY}

Luisa L. Orduz-Díaz ${ }^{1}$ Freddy A. Bernal ${ }^{1}$ Ericsson D. Coy-Barrera ${ }^{1,2}$

\section{RESUMEN}

La pteridina reductasa-1 (PTR1) ha sido descubierta como la responsable de la reducción de la susceptibilidad a antifolatos (e.g., metotrexato) de parásitos tripanosómicos, lo cual la ha convertido en un objetivo quimioterapeútico. Por esta razón, trece diterpenos conocidos, de núcleo kaurano, fueron evaluados in silico contra la PTR1, mediante docking molecular y modelamiento farmacofórico. El docking molecular mostró claras interacciones polares con algunos residuos del sitio activo de la PTR1, que dependieron principalmente de la presencia de un grupo carboxilo en C19. Estos resultados fueron corroborados por el mapeo de interacciones residuales, indicando que los ácidos kauren-19-oicos poseen las características estructurales importantes para una inhibición de la PTR1, lo cual es un excelente punto de partida para futuros estudios de optimización estructural de este tipo de compuestos.

Palabras clave: Diterpenos, kaurano, in silico, docking molecular.

1 Laboratorio de Química Bioorgánica, Departamento de Química, Facultad de Ciencias Básicas y Aplicadas, Universidad Militar Nueva Granada, Cajicá, Colombia.

2 Autor para correspondencia: ericsson.coy@unimilitar.edu.co 


\section{ABSTRACT}

Pteridine reductase-1 (PTR1) is one of enzymes discovered as being responsible for the reduced susceptibility to antifolates (eg, metotrexato) of trypanosomic parasites, which has become a chemotherapeutic target. Therefore, thirteen known kaurane-related diterpenes were evaluated in silico against PTR1 by molecular docking and pharmacophore modeling. The molecular docking exhibited clear polar interactions with some active site residues of PTR1, which was primarily dependent on the presence of a carboxyl group at C19. These results were supported by the pharmacophore modeling, indicating that kauren-19-oic acids possess significant structural features for inhibition of PTR1, which is an excellent starting point for future studies of structural optimization of this kind of compounds.

Keywords: Diterpenes, kaurane, in silico, molecular docking.

\section{INTRODUCCIÓN}

Los parásitos del género Leishmania son protozoos tripanosomátidos causantes de la enfermedad desatendida conocida como la Leishmaniosis, la cual causa infección de 15 millones de personas alrededor del mundo bajo sus tres formas clínicas: cutánea, mucocutánea y visceral (Loría-Cervera y AndradeNarváez, 2014; Reithinger et al., 2007). Su emergencia como un patógeno oportunista ha generado un interés en salud pública y una necesidad recurrente de controlarla. No obstante, su tratamiento continúa siendo basado en el uso de sales de antimonio pentavalente como medicamentos de primera línea, o el uso de la amfotericina B y pentamidina, como medicamentos de segunda línea, los cuales son frecuentemente tóxicos, algunos tienen un modo de acción desconocido, y usualmente son marginalmente efectivos, sumado al agravante que ya hay diversos brotes de resistencia (Arboleda et al., 2013; Sundar y Rai, 2002). A la fecha, no han sido muchos los avances en la sustitución de estos medicamentos, con algunos casos de terapia inciertamente eficaces (Pham et al,
2013), aunque ya existen algunos en ensayos clínicos basados en medicamentos ya conocidos, tales como el allopurino ${ }^{\circledast}$ (inhibidor de síntesis de proteínas), el ambisoma ${ }^{\circledR}$ (una formulación de amfotericina B en liposomas) y el ketaconazo ${ }^{\circledR}$ (un inhibidor de la síntesis de esteroles) (Jebran et al., 2014; Setzer, 2013).

Una ruta metabólica que ha sido explotada para el tratamiento de enfermedades parasitarias involucra la biosíntesis de co-factores de tipo folato a través de la inhibición de la dihidrofolato reductasa (DHFR) (Nare et al., 1997). Sin embargo, los parásitos del género Leishmania son organismos auxótrofos para el folato, lo que significa que ellos poseen una completa y sofisticada ruta metabólica para resguardar pteridinas del hospedero e incorporarlo a algún metabolismo intermediario y/o alternativo (Ong et al., 2011). Entre esas alternativas, se conoce que los parásitos de Leishmania toman pterinas (como la biopterina), las cuales sufren reducciones sucesivas para formar tetrahidroderivados que son activos. Una de las dos enzimas conocidas para Leishmania que 
puede catalizar estas reducciones es la pteridina reductasa (PTR1), que transforma pterinas conjugadas y no-conjugadas, como por ejemplo la reducción de biopterina a dihidrobioterina, y luego a tetrahidrobiopterina (Esquema 1) (Ong et al., 2011), cuya reacción es altamente importante para resguardar y mantener cantidades intracelulares vitalmente importantes de tetrahidropterina y ha sido probada como parte esencial del crecimiento in vivo mediante estudios de expresión de genes (Kheirandish et al., 2012). Debido a que la PTR1 es menos sensible al efecto del metotrexato, pero cataliza también la reducción de folatos, esto explica las fallas terapeúticas de este tipo de fármacos contra parásitos tripanosomátidos (Ong et al., 2011).
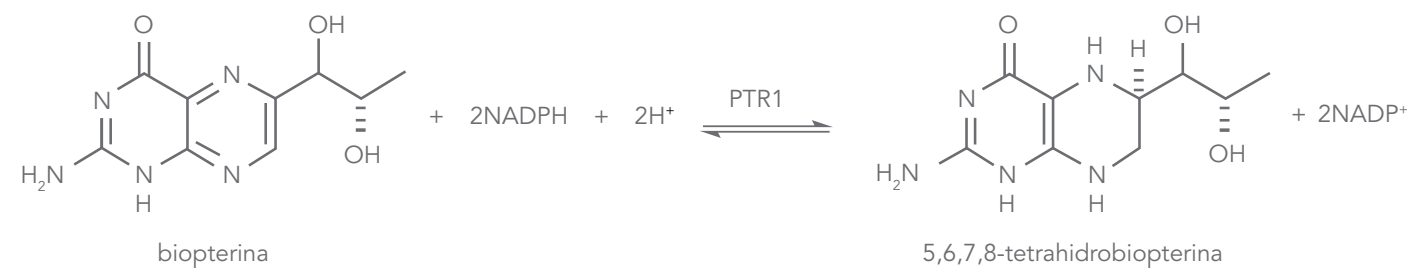

Esquema 1. Reacción de producción de tetrahidropterinas catalizada por PTR1

Varios diterpenos aislados de fuentes naturales han mostrados efectividad in vitro contra promastigotes y amastigotes multiresistentes de Leishmania (Singh et al., 2014; Santos et al., 2013; Kennedy et al., 2011; Jullian et al., 2005). Sin embargo, su modo de acción no ha sido dilucidado y la posible actividad contra la PTR1 permanece inexplorada. Por tanto, como parte de nuestro interés en la búsqueda de nuevos prototipos contra la leishmaniosis, en el presente trabajo se describe el estudio realizado sobre la base molecular a nivel in silico de trece diterpenos de nucleo kaurano contra la PTR1, mediante docking molecular y modelamiento farmacofórico. Estos análisis mostraron detalles muy importantes a nivel molecular hacia el desarrollo de prototipos terapeúticos basados en diterpenos.

\section{PARTE EXPERIMENTAL}

\section{Modelamiento molecular a nivel DFT}

Los compuestos evaluados fueron seleccionados debido a sus reportes en diferentes estudios previos (Singh et al., 2014). Una búsqueda conformacional aleatoria Monte-Carlo, sin ningún tipo de restricciones geométricas, se hizo mediante parametrización semi-empírica AM1 (Dewar et al., 1985) incluido en el software SPARTAN con un límite de 500 confórmeros y utilizando el campo de fuerza molecular (MMFF) de Merck implementado en el paquete del software. Los confórmeros energéticamente más estables, dentro de un rango energético de $6 \mathrm{kcal} / \mathrm{mol}$, fueron optimizados a nivel DFT mediante el uso de la combinación del funcional B3LYP y el grupo de bases 6-31+G* (Becke, 1993). Después de la optimización DFT, un conjunto de conformaciones (con energías no más de aproximadamente $3 \mathrm{kcal} / \mathrm{mol}$ por encima de la estructura energéticamente más baja) se consideraron para el análisis de la población de Boltzmann. Las conformaciones restantes fueron descartadas porque estaban duplicadas o tenían mucha energía. Por lo tanto, de acuerdo con el análisis de la población de Boltzmann, se obtuvieron entonces los confórmeros más estables.

\section{Análisis por Docking}

Los experimentos de docking molecular se realizaron con el plug-in Autodock/Vina (1.1.2) para 
PyMOL (1.3r2) bajo entorno Python 2.5.2 en Windows (Seeliger y de Groot, 2010). Las coordenadas de la estructura cristalina de Rayos-X de la enzima PTR1 se obtuvieron del Protein Data Bank RCSB (Código PDB: 2QHX). A la enzima se le agregaron los átomos de hidrógeno y las moléculas de agua. Las moléculas de ligando se optimizaron como se describió en la sección anterior. El experimento de acoplamiento sobre la PTR1 se llevó a cabo entre el ligando-minimizado en la enzima $2 \mathrm{OHX}$ a través de un cubo en el centro geométrico del ligando nativo presente en la estructura PDB evaluada, con las dimensiones $24 \times 24 \times 24 \AA$, que cubre el sitio de unión del ligando con un espaciado de rejilla de 0,375 A. Las poses de acoplamiento se clasificaron de acuerdo a sus puntajes de docking (como la energía libre de acoplamiento o afinidad) y tanto la lista clasificada de ligandos acoplados y sus correspondientes poses de docking se exportaron como un archivo CSV para su posterior análisis. Los valores de RMSD no superaron los 1,2 Å. Como control del estudio in silico se utilizó el inhibidor 1-[4-[(2,4-diaminopteridin-6-il)metil-metilamino] benzoil]piperidina-4-carboxilato de metilo (DAMBPC) (Cavazzuti, et al., 2008).

\section{Modelamiento Farmacofórico}

El modelamiento farmacofórico se realizó en el programa Discovery Studio 2.0 (Accelrys ${ }^{\circledast}$, San Diego, USA). En este programa las moléculas se importaron separadamente como archivos .mol2 previamente optimizadas a nivel DFT. Una vez importada, se definieron las características moleculares de cada una a través del algoritmo para modelamiento farmacofórico del software, identificando los centros de los farmacóforos posibles. Una vez el modelo fue obtenido, la molécula se acopló nuevamente al sitio activo de la enzima PTR1, para luego identificar si los farmacóforos descritos influían en las interacciones residuales del complejo en ese sitio activo. Por otro lado, el grupo total de moléculas fue alineado con el algoritmo de alineación incluido en el paquete, y luego se agruparon mediante análisis de componentes principales (ACP) con el fin de identificar similitudes en los perfiles moleculares.

\section{RESULTADOS Y DISCUSIÓN}

Las estructuras de los compuestos evaluados se muestran en la Figura 1, las cuales fueron separadamente optimizadas a nivel DFT/B3LYP/6-31G* (Dewar et al., 1985). Como era de esperarse, los confórmeros más estables obtenidos para las estructuras 1-13 exhibieron conformaciones de sillas para los anillos A y B. Así mismo, el fragmento biciclo[3.2.1] octánico de todos los diterpenos resultó sin distorsiones evidentes a pesar de sus funcionalizaciones, con ejes de simetría entre los carbonos C11 y C14.

El confórmero más estable de cada estructura optimizada fue importado a PyMOL para ser acoplada al sitio activo de la enzima PTR1. La estructura cristalina de la enzima fue obtenida del Protein Data Bank (Código PDG: 2QHX), cuya estructura tridimensional se observa en la Figura 2.

\section{Entre esas alternativas, se conoce que los parásitos de Leishmania toman pterinas (como la biopterina), las cuales sufren reducciones sucesivas para formar tetrahidroderivados que son activos.}




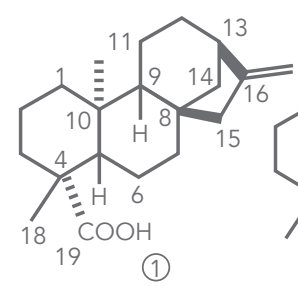

(1)

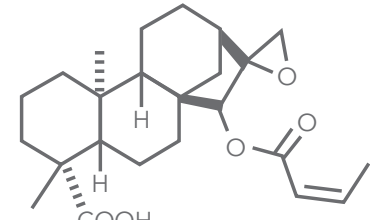

(6)

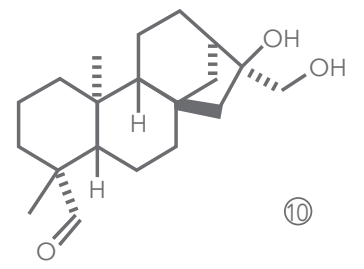

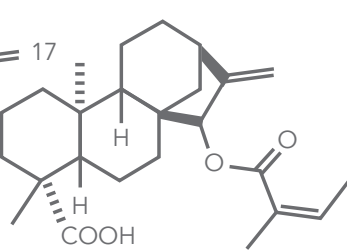

(2)

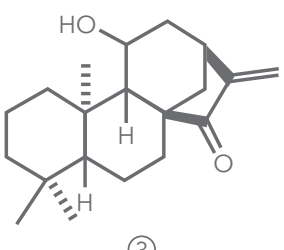

(3)

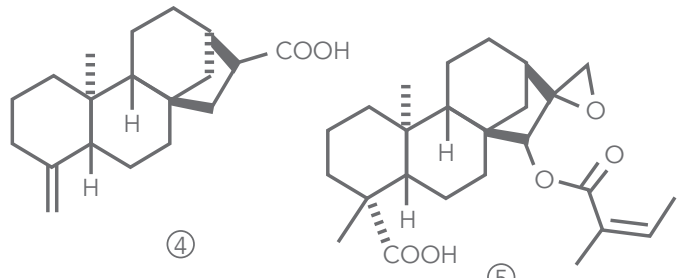

(5)

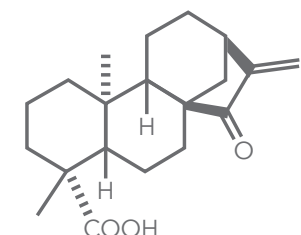

(7)

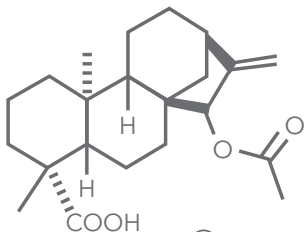

(8)

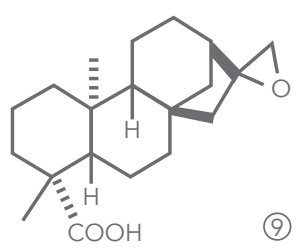

(9)

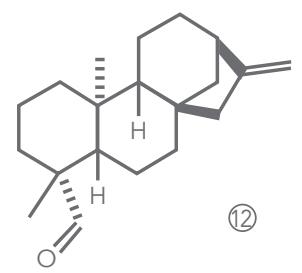

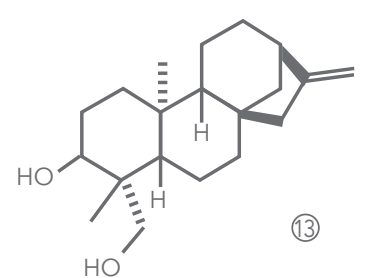

Figura 1. Estructuras de los diterpenos de núcleo kaurano.

El sitio activo evaluado se compone principalmente de los residuos Ser111, Phe113 y Tyr191, los cuales fueron adoptados de acuerdo a lo publicado por Cavazzuti, et al., quiénes los reportan como residuos activos donde la interacción pterina $\cdots$ enzima se concibe (Cavazzuti, et al., 2008). A través del plugin Autodock/Vina en ambiente Python 2.5 (Seeliger y de Groot, 2010), se realizó el docking molecular de los trece compuestos. Como control se incluyó el compuesto 1-[4-[(2,4-diaminopteridin-6-il)metil-metilamino]benzoil]piperidine-4-carboxilato de metilo (DAMBPC), el cual ha sido reportado como un excelente inhibidor de la PTR1 (Cavazzuti, et al., 2008).

En la Tabla No 1 se muestran los resultados de afinidad para la primera pose en la interacción ligando...enzima para los diterpenos evaluados. Estos datos muestran que el diterpeno 5 manifiesta una mejor afinidad $(-9,7 \mathrm{kcal} / \mathrm{mol})$ que el inhibidor DAMPBC, lo cual resulta altamente relevante desde

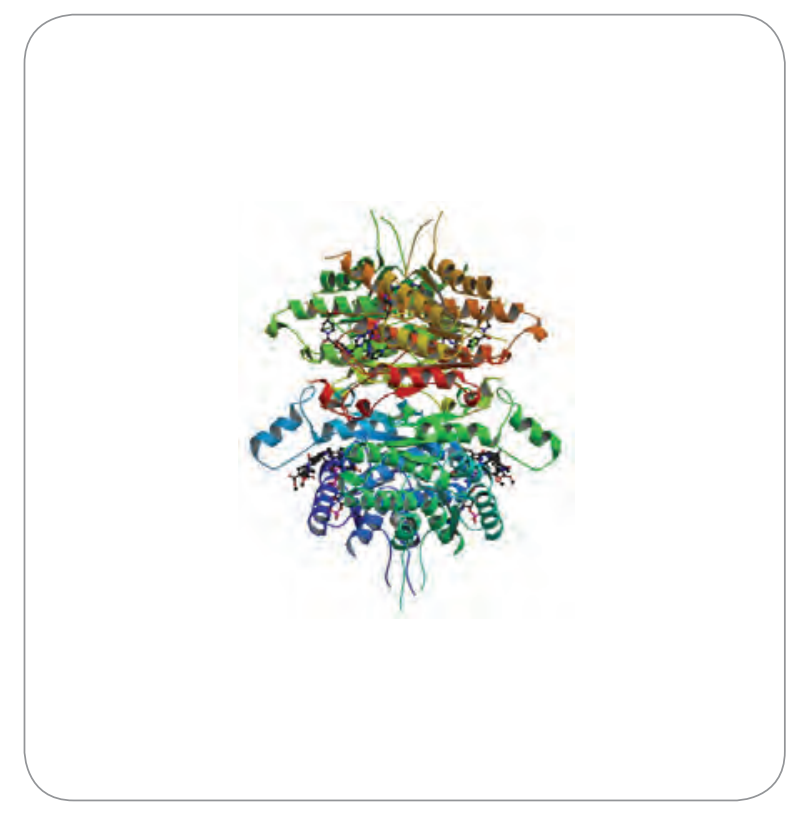

Figura 2. Estructura tridimensional de la PTR1 (tomada del PDB, www. rcsb.org, código $2 \mathrm{QHX}$ ) 
el punto de vista farmacológico. Así mismo, los compuestos 2 y 6 exhibieron afinidad muy cercana al control $(-9,4 \mathrm{kcal} / \mathrm{mol})$. Este resultado se constituye en un punto de partida importante para tomar a estos diterpenos como moléculas plantilla con el fin de realizar futuros estudios de optimización estructural y de esta manera mejorar su actividad en todas aquellas etapas del proceso (in silico, in vitro e in vivo). Igualmente, los compuestos 2 y 5 poseen estructuras muy similares (diferenciándose solamente por la presencia del grupo epóxido en los carbonos C16 y $\mathrm{C} 17$ en 6) resultando en afinidades muy similares

Tabla 1. Puntajes Vina (Afinidad) resultantes del Docking molecular de la PTR1 con cada uno de los diterpenos evaluados.

\begin{tabular}{ccc} 
Clasificación $^{\text {a }}$ & Compuesto & $\begin{array}{c}\text { Afinidad }^{b} \\
\text { (kcal/mol) }\end{array}$ \\
\hline 1 & 5 & $-9,7$ \\
\hline 2 & 6 & $-9,4$ \\
\hline 3 & 2 & $-9,4$ \\
\hline 4 & 8 & $-9,2$ \\
\hline 5 & 7 & $-8,8$ \\
\hline 6 & 9 & $-8,7$ \\
\hline 7 & 1 & $-8,7$ \\
\hline 8 & 3 & $-8,6$ \\
\hline 9 & 10 & $-8,5$ \\
\hline 10 & 4 & $-8,5$ \\
\hline 11 & 13 & $-8,4$ \\
\hline 12 & 12 & $-8,2$ \\
\hline 13 & 11 & $-8,0$ \\
\hline control & DAMBPC & $-9,5$
\end{tabular}

${ }^{a}$ Clasificación según pose de mayor afinidad; ${ }^{b}$ Como puntajes Vina obtenidos del Docking molecular; ' ${ }^{\mathrm{DAMMBC}}=1$-[4-[(2,4-diaminopteridin6-il)metil-metilamino]benzoil]piperidina-4-carboxilato de metilo, inhibidor de la PTR1 utilizado como control (Cavazzuti, et al., 2008).
$(-9,4 \mathrm{vs}-9,7 \mathrm{kcal} / \mathrm{mol}$, respectivamente), lo cual indica que la presencia o ausencia del grupo epóxido no es un factor que aporte al acoplamiento del ligando a la enzima. Lo anterior es confirmado con las afinidades obtenidas para los compuestos 1 y 9 (ambas de -8,7 $\mathrm{kcal} / \mathrm{mol})$, cuya diferencia estructural es la misma: un grupo epóxido en C17-C16 en 9.

En la Figura No 3 se observan los complejos con las poses con mayor afinidad de los diterpenos luego del acoplamiento con la PTR1, donde se evidencian los detalles estructurales que proporcionan información sobre la forma del acoplamiento. Por ejemplo, se aprecia que los diterpenos que poseen un grupo carboxilo en C19 generan tres contactos polares de tipo puente de hidrógeno con los residuos Ser111 y Phe113, estabilizando convenientemente el complejo ligando...enzima. Las distancias de los puentes de hidrógeno se mantuvieron en una media de $\sim 2,0$,

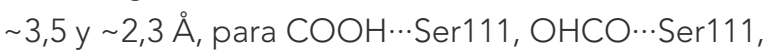
y $\mathrm{OHCO}$...Phe113, respectivamente.

Estos resultados coinciden con la clasificación obtenida por los valores de afinidad, cuyas siete primeras clasificaciones corresponden a compuestos que cumplen con este requerimiento estructural. Adicionalmente, los resultados reflejaron otra exigencia funcional para estabilizar aún más el complejo enzima...ligando, la cual corresponde a la presencia de un grupo angeloil, senecioil o acetil en la posición 15 (con orientación $\beta$; el epímero $\alpha$ no fue considerado en el presente estudio). No obstante, al parecer la presencia del éster (o algún grupo polar) en la posición 15, ocasiona que el ligando no pueda acercarse a los residuos Ser111 y Phe113, pero dada la interacción hidrofóbica con la parte aromática de la Phe113 y de la Tyr191, se origina una mejor estabilización del complejo.

Por otro lado, los ligandos con grupos $\mathrm{OH}$ en el anillo $A$, presentaron igualmente un puente de hidrógeno con la Phe113 (2,2 A), pero resultando en una afinidad menor. Lo anterior indica que los grupos 

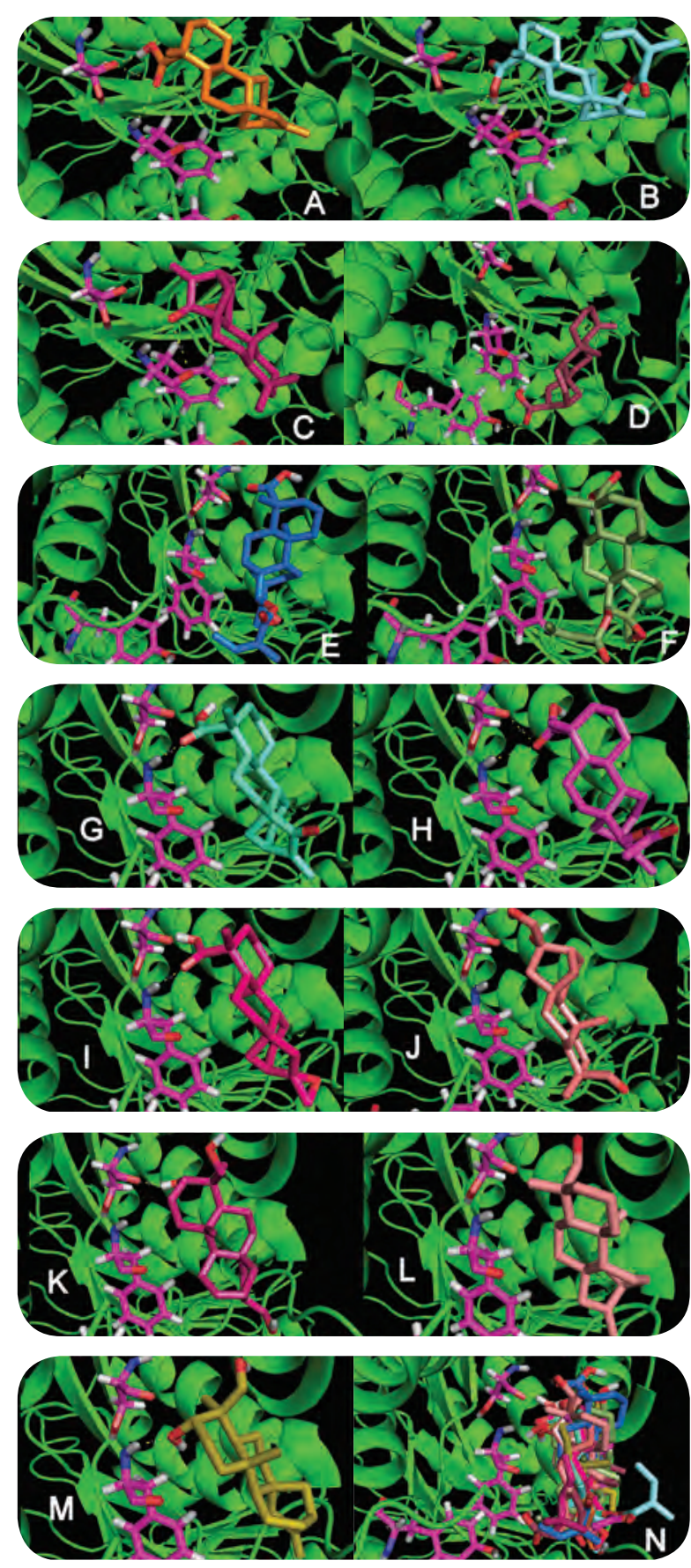

Figura 3. Complejos con las poses con mayor afinidad de los diterpenos luego de acoplarse con la PTR1. Imágenes A-M corresponden a complejo ligando $\cdots$ enzima: A) $1 \cdots$ PTR1; B) $2 \cdots$ PTR1; C) $3 \cdots$ PTR1; D)

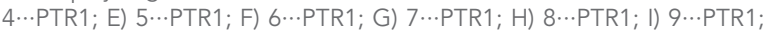
J) $10 \cdots$ PTR1; K) $11 \cdots$ PTR1; L) $12 \cdots$ PTR1 M) $13 \cdots$ PTR1; N) La pose con mayor afinidad de todos los ligandos 1-13 con la enzima.

ISSN 1900-4699 • Volumen 9 • Número 1 • Páginas 142-153 • 2013 polares son importantes durante el docking en el sitio activo de la PTR1. Sin embargo, es posible apreciar que el grupo carboxílico proporciona una mayor estabilidad que otros grupos polares en este mismo anillo. Éste hecho está sujeto a la posibilidad de realizar estudios futuros de evaluación de otras estructuras con grupos carboxilo en diferentes posiciones del anillo con fines de diseño racional y optimización.

Un resultado particular ocurrió con el compuesto 4, cuya pose con mejor afinidad fue aquella que presentaba un puente de hidrógeno $(1,5 \AA$ A ) del grupo carboxilo en la posición 16 con el grupo $\mathrm{OH}$ de la Tyr191. Esto permite nuevamente deducir que el grupo carboxilo es un requisito estructuralmente esencial para inhibidores de la PTR1 bajo interacción polar con los residuos del sitio activo, con la ayuda de contactos hidrofóbicos en la zona media del ligando con la Phe113.

Para indagar más sobre los requerimientos estructurales encontrados para los ligandos evaluados, se realizó el modelamiento farmacofórico para los diterpenos más activos, cuyos modelos son mostrados en la Figura 4. Allí es posible apreciar que los compuestos con grupo carboxilo en el aniIlo A (como 1, 3, 5) poseen la característica determinante para la interacción con la Ser111 y la Phe113 que corresponde a la orientación de los vectores del donor (grupo $\mathrm{C}=\mathrm{O}$ ) y del aceptor (grupo $\mathrm{OH}$ ) de puentes de hidrógeno en el grupo carboxilo, cuya distancia y ángulo diedro son apropiados para interactuar con los puntos polares de estos residuos en el sitio activo $\left(2,3 \AA\right.$ y $37^{\circ}$, respectivamente). Esta disposición, por consiguiente, permite que exista la ya mencionada estabilización adecuada del complejo. Sin embargo, como se observó en los resultados del docking, otro requisito estructural es la presencia de contactos hidrofóbicos en la parte media de la molécula para la interacción con el anillo aromático de la Phe113, lo cual es representado notoriamente en los modelos farmacofóricos 
(Figura 3). Sin embargo, el compuesto 1, el ácido kaurenoico, fue el diterpeno con grupo carboxilo en el anillo A que menor valor de afinidad presentó. Comparándolo con los modelos de los compuestos 5 y 6 (los más afines, con $-9,7$ y $-9,4 \mathrm{kcal} / \mathrm{mol}$ ), en el extremo bicíclico se encuentra un centroide hidrofóbico generado por el doble enlace, $\Delta^{16}$. No obstante, los compuestos 5 y 6 , a diferencia de 1 , y aparte del grupo epóxido que al parecer no es un grupo aportante en el docking, tienen grupos angeloil/senecioil, respectivamente, como ésteres en C15, cuyo aceptor de puentes de hidrógeno debe estar promoviendo estabilización del complejo mediante contactos polares, generando mejores afinidades. Esto último es demostrado por los resultados de docking de los compuestos 7 y 8 , los cuales poseen estructura similar a 2, pero a diferencia poseen en $\mathrm{C} 15$ un grupo oxo y un acetilo, respectivamente. Estos compuestos 8 y 7 , se clasificaron como los diterpenos $4^{\circ}$ y $5^{\circ}$ con mayor afinidad, respectivamente, después de los diterpenos 5,6 y 2 . Estos cinco primeros compuestos clasificados por afinidad, comparten el hecho de poseer el grupo carboxilo en 19 y un grupo aceptor en C15. Por tanto, se podría indicar que los diterpenos con núcleo kaurano candidatos a ser inhibidores de la PTR1 deben poseer como farmacóforos un grupo carboxilo (i.e., un grupo combinado con donor/aceptor), una sección media que permita contactos hidrofóbicos y un grupo aceptor en C15.

En el marco de estas observaciones, se realizó un análisis in silico de la interacción ligando-enzima

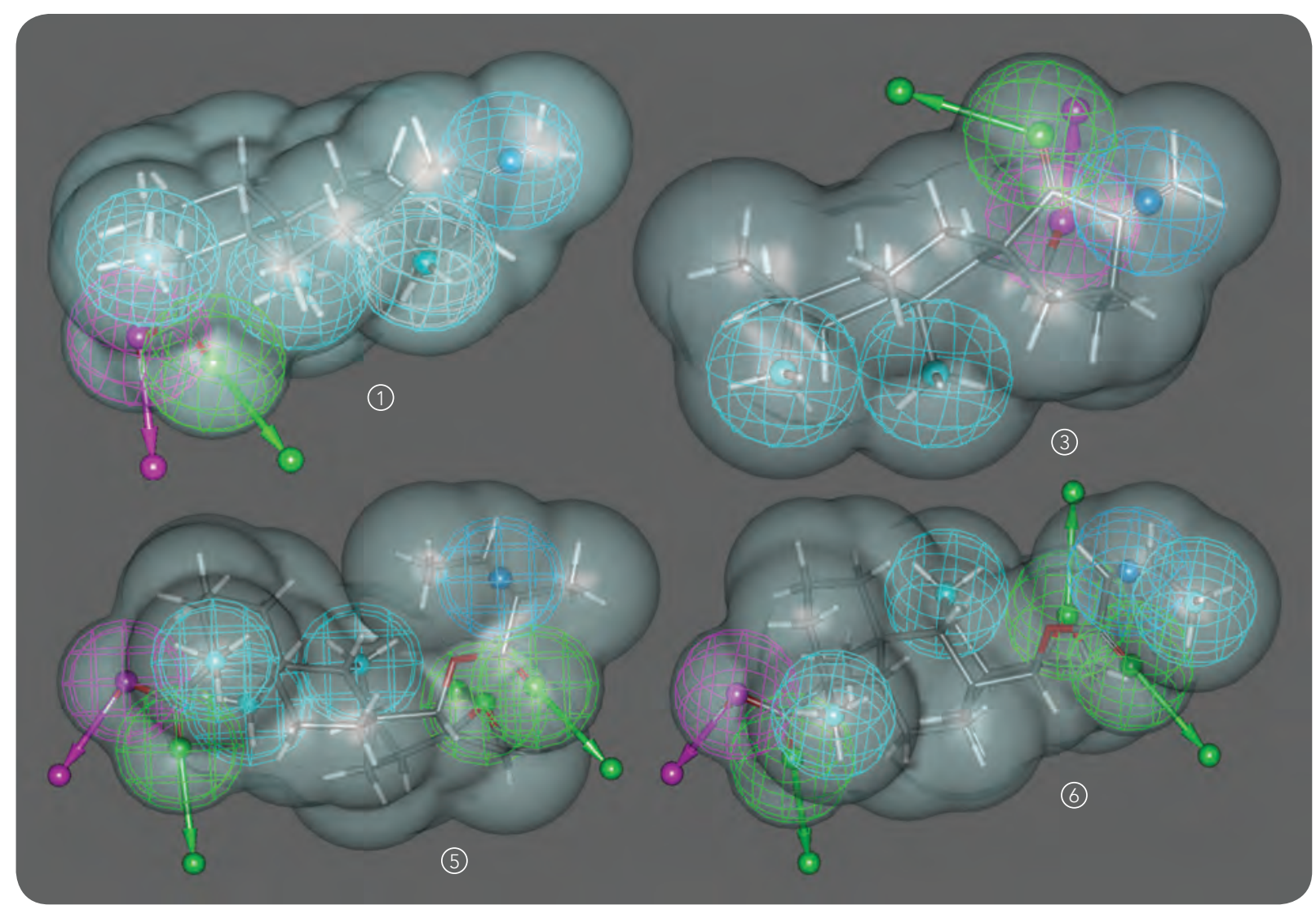

Figura 4. Modelos farmacofóricos de los compuestos 1, 3, 5, y 6 . 
para el diterpeno más activo, 5, el de núcleo base, 1 , y el control, DAMBPC, dando como resultado un mapa 2D de las interacciones residuales de estos ligandos con la enzima PTR1, lo cual es mostrado en la Figura 5. En estos mapas, el compuesto control evidenció una serie larga de asociaciones con varios residuos (Figura 5C), debido a la longitud de la molécula en su conformación más estable. Aquí se destaca el anclaje hidrofóbico ח-ா de la amida aromática con la Arg17 y un puente de hidrógeno con el grupo amino primario del anillo pteridinona con la Asp142. Tales interacciones no se apreciaron para los otros dos compuestos 1 y 5 . Es de anotar que los residuos reportados para el sitio activo, Ser111, Phe113 y Tyr191, están considerados en este mapa, los cuales están ubicados alrededor del grupo p-aminobenzilamida, pero sin interacción directa aparente, salvo el extremo polar de la Phe113, que está en contacto leve con el fragmento p-aminobenzilamida.

Para el caso del compuesto base, 1, (Figura 5A), se ve directamente que lo obtenido por docking

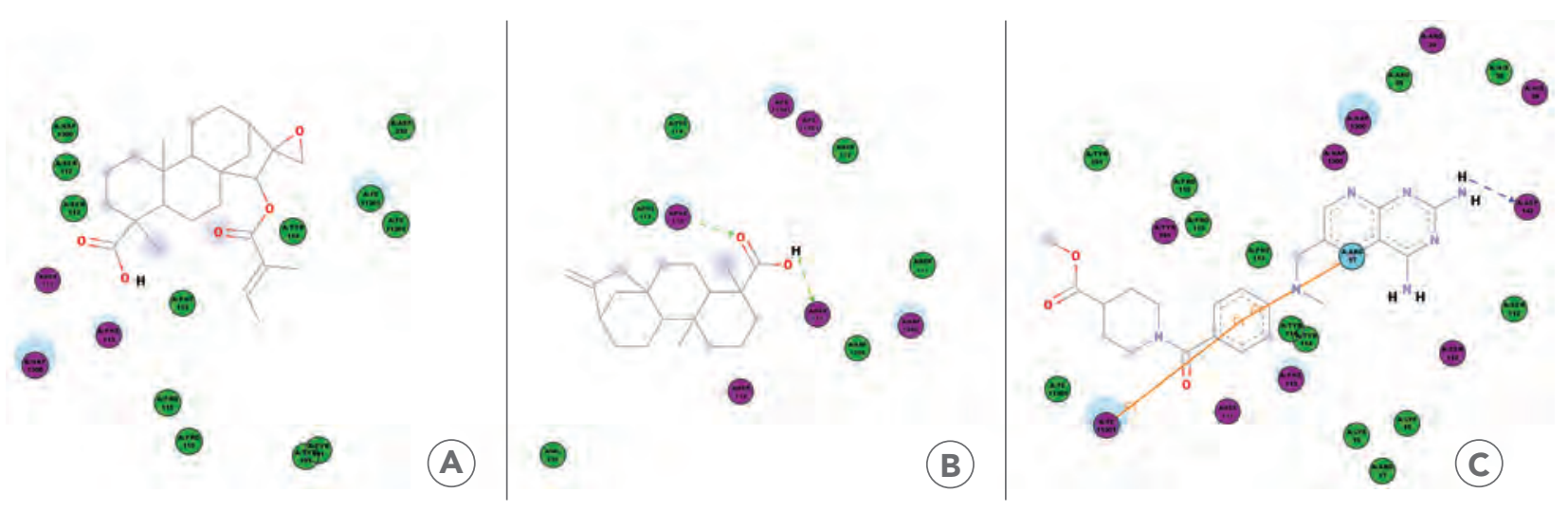

Figura 5. Interacción ligando-enzima para los compuestos A) 5, B) 1 y C) DAMBPC.

resulta de la asociación polar del grupo carboxilo en C19 con la Ser111 y la Phe113, pero que, debido al extremo hidrofóbico del anillo $\mathrm{B}$ y del biciclooctano, las interacciones residuales son más bien bajas. En cambio, el compuesto con mayor afinidad, 5, evidencia una serie de contactos que apoyan lo indicado en el modelo farmacofórico, evidenciando que el extremo carboxílico en C19 mantiene una unión polar con la Ser111 y la Phe113, y que la parte central esta interactuando con el fragmento hidrofóbico de la Phe113. Adicionalmente, este mapa de interacciones sugiere que el aceptor requerido en $\mathrm{C} 15$ (percibido también en el mapa respectivo con los otros compuestos; datos no mostrados) debe estar posiblemente generando un contacto polar con la Tyr114, no considerada anteriormente como crucial en el sitio activo, pero que, según los resultados obtenidos, al parecer es importante para el acoplamiento de este tipo de diterpenos.

Finalmente, para confirmar que los farmacóforos identificados en el presente estudio corresponden a los responsables de la afinidad de los diterpenos con la PTR1 y la posible actividad observada para los mismos contra Leishmania, un agrupamiento obtenido por análisis de componentes principales (ACP) fue realizado utilizando los perfiles moleculares de cada una de las estructuras después de su alineación, para luego ser contrastada con los resultados de afinidad del docking, dando como resultado el diagrama de correlación de la Figura 6. En este diagrama se puede evidenciar la similitud encontrada para los compuestos 5 y 6 , que corresponden a los 
más afines; y para los compuestos 2 y 8, que son los siguientes más activos, cuyas agrupaciones por similitud se encuentran en el mismo cuadrante del diagrama. Un tercer agrupamiento, ubicado en otro cuadrante, corresponde a compuestos que poseen bajas similitudes moleculares (compuestos 1, 3, 4, 7, 12) cuyo valor de afinidad también es muy alto (>$8,8 \mathrm{kcal} / \mathrm{mol}$ ), seguido de otros compuestos, ubicados en los restantes cuadrantes, que exhibieron similitudes casi nulas $(9,11,13,10)$. Todo lo anterior corrobora lo observado en el docking molecular, relacionado con la presencia de un perfil molecular característico, basado en un núcleo kaurano, con grupo carboxilo en C19 y un aceptor en C15.

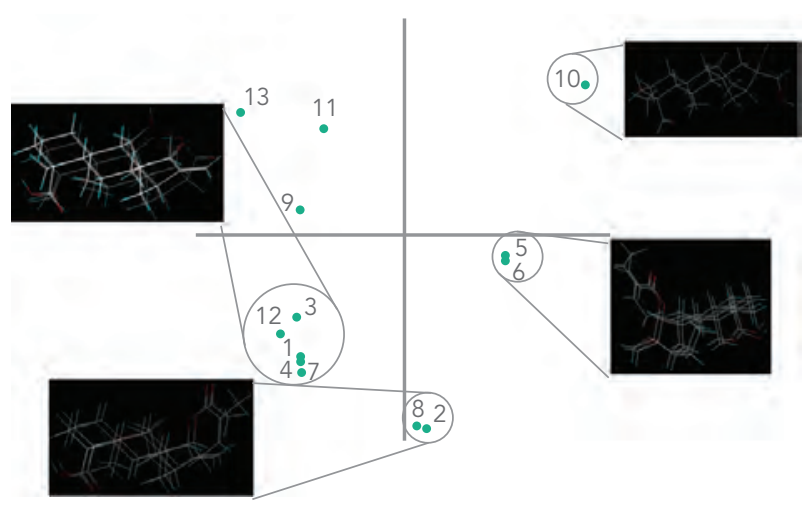

Figura 6. Agrupamiento por ACP derivado de similitudes de perfiles moleculares.

\section{CONCLUSIONES}

La PTR1 es un objetivo farmacológico preponderante en quimioterapia para el control de la Leishmaniosis. Por tanto, es altamente requerido el estudio racional de nuevos prototipos que sirvan de modelo para el desarrollo de terapias eficaces y seguras para este fin. Dado que los diterpenos son compuestos que han presentado actividad contra los dos estadios de parásitos del género Leishmania, en el presente estudio se evaluaron 13 compuestos con estructura diterpénica a nivel in silico contra la PTR1. A través del docking molecular se encontró que los diterpenos con grupo carboxilo en C19 favorecen un contacto polar tipo puente de hidrógeno con los residuos Ser111 y Phe113 y una interacción hidrofóbica también con e I residuo Phe 113. Debido al análisis de los modelos farmacofóricos y el mapa de interacciones residuales, se pudo establecer que el perfil molecular de los diterpenos debe estar basado principalmente por un grupo carboxilo (o un grupo combinado con donor/aceptor), una sección intermedia que permita contactos hidrofóbicos y un grupo aceptor en C15, como farmacóforos. Así mismo, se encontró en el mapa de interacciones residuales que el requerimiento estructural basado en el grupo aceptor en C15 puede estar condicionado por una asociación con la Tyr114, un residuo en el sitio activo no considerado previamente, pero que resulta significativo para la interacción ligando...enzima de diterpenos con núcleo kaurano. El presente estudio se constituye como un excelente punto de partida para futuros estudios de optimización estructural de este tipo de compuestos, cuyos detalles observados a nivel molecular permiten abrir una ruta de trabajo hacia el desarrollo de prototipos terapeúticos contra Leishmania basados en diterpenos con núcleo kaurano.

\section{AGRADECIMIENTOS}

El presente estudio corresponde a un producto derivado del proyecto CIAS-1172 financiado por la Vicerrectoría de Investigaciones de la Universidad Militar Nueva Granada - Vigencia 2013. 


\section{BIBLIOGRAFÍA}

1. Arboleda M, Jaramillo L, Ortiz D, Díaz A. 2013. Leishmaniasis cutáne y herpes zoster multidermatómico. Revista chilena de infectología, 30:680-682.

2. Becke AD. 1993. Density-Functional Thermochemistry. III. The role of exact exchange. Journal of Chemical Physics, 98:5648-5652.

3. Cavazzuti A, Paglietti G, Hunter WN, Gamarro F, Piras S, Loriga M, Allecca S, Corona P, McLuskey K, Tulloch L, Gibellini F, Ferrari S, Costi MP. 2008. Discovery of potent pteridine reductase inhibitors to guide antiparasite drug development. Proceedings of the National Academy of Sciences USA. 105:1448-1453.

4. Dewar M, Zoebisch E, Healy E, Stewart J. 1985. AM1: a new general purpose quantum mechanical molecular model. Journal of American Chemical Society, 107:3902-3909.

5. Jebran $A F$, Schleicher $U$, Steiner $R$, Wentker $P$, Mahfuz F, Stahl HC, Amin FM, Bogdan C, Stahl KW. 2014. Rapid Healing of Cutaneous Leishmaniasis by High-Frequency Electrocauterization and Hydrogel Wound Care with or without
DAC N-055: A Randomized Controlled Phase Ila Trial in Kabul. PLOS Neglected Tropical Diseases, 8:e2694.

6. Jullian V, Bonduelle C, Valentin A, Acebey L, Duigou AG, Prévost MF, Sauvain M. 2005. New clerodane diterpenoids from Laetia procera (Poepp.) Eichler (Flacourtiaceae), with antiplasmodial and antileishmanial activities. Bioorganic \& Medicinal Chemistry Letters, 15:5065-5070.

7. Kennedy ML, Llanos GG, Castanys S, Gamarro F, Bazzocchi IL, Jiménez IA. 2011. Terpenoids from Maytenus species and assessment of their reversal activity against a multidrug-resistant Leishmania tropical line. Chemistry \& Biodiversity, 8:2291-2298.

8. Kheirandish F, Bandehpour M, Haghighi A, Mahboudi F, Mohebali M, Kazemi B. 2012. Inhibition of Leishmania major PTR1 Gene Expression by Antisense in Escherichia coli. Iranian Journal of Public Health. 41:65-71.

9. Loría-Cervera EN, Andrade-Narváez FJ. 2014. Animal models for the study of leishmaniasis 
immunology. Revista do Instituto de Medicina Tropical de São Paulo, 56:1-11.

10. Nare B, Hardy LW, Beverley SM. 1997. The roles of pteridine reductase 1 and dihydrofolate reductase-thymidylate synthase in pteridine metabolism in the protozoan parasite Leishmania major. The Journal of Biological Chemistry, 272:13883-13891.

11. Ong HB, Sienkiewicz N, Wyllie S, Fairlamb AH. 2011. Dissecting the metabolic roles of pteridine reductase 1 in Trypanosoma brucei and Leishmania major. The Journal of Biological Chemistry, 286:10429-10438.

12. Pham TT, Loiseau PM, Barratt G. 2013. Strategies for the design of orally bioavailable antileishmanial treatments. International Journal of Pharmaceutics, 454:539-52.

13. Reithinger R, Dujardin JC, Louzir H, Pirmez C, Alexander B, Brooker S. 2007. Cutaneous leishmaniasis. The Lancet Infectious Diseases, 7:581-596.

14. Santos AO, Izumi E, Ueda-Nakamura T, DiasFilho BP, Veiga-Júnior VF, Nakamura CV. 2013.
Antileishmanial activity of diterpene acids in copaiba oil. Memórias do Instituto Oswaldo Cruz, 108:59-64.

15. Seeliger D, de Groot BL. 2010. Ligand docking and binding site analysis with PyMOL and $\mathrm{Au}$ todock/Vina. Journal of Computer-Aided Molecular Design, 24:417-422.

16. Setzer WN. 2013. Trypanosomatid disease drug discovery and target identification. Future Medicinal Chemistry, 5:1703-1704.

17. Singh N, Mishra BB, Bajpai S, Singh RK, Tiwari VK. 2014. Natural product based leads to fight against leishmaniasis. Bioorganic \& Medicinal Chemistry. 22:18-45.

18. Sundar S, Rai M. 2002. Advances in the treatment of leishmaniasis. Current Opinion in Infectious Diseases, 15:593-598. 